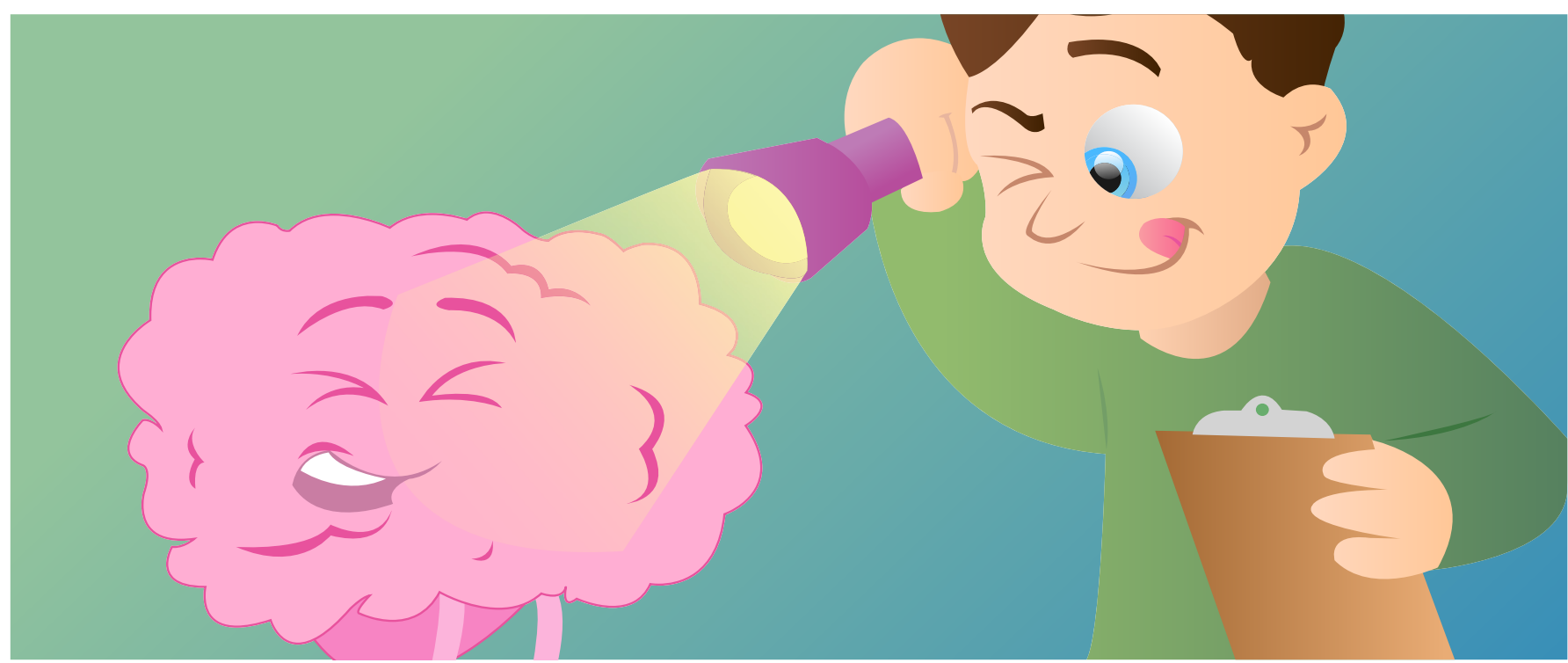

\title{
WHAT IS OPTOGENETICS AND HOW CAN WE USE IT TO DISCOVER MORE ABOUT THE BRAIN?
}

\section{Diana H. Lim ${ }^{1 *}$ and Jeffrey LeDue ${ }^{2}$}

${ }^{1}$ Department of Psychology, Concordia University, Montreal, QC, Canada, ²Djavad Mowafaghian Centre for Brain Health, University of British Columbia, Vancouver, BC, Canada

\section{REVIEWED BY: \\ PRUTHVI 15 YEARS OLD}

\section{NEURONS}

Special cells in the brain communicate with each other by sending and receiving electrical and chemical signals. There are billions of neurons in the brain, and the signals sent between these cells are the basis for all of our thoughts and behaviors. Neurons are sometimes called nerve cells.
How does the brain work? This is a question that scientists have been interested in for hundreds of years. In order to figure out how the brain works, scientists have had to do lots of experiments and figure out ways to examine and test the brain. In 2005, a new technique was created, called optogenetics. This technique uses a combination of light and genetic engineering (changing the genetic information of a living thing by inserting or deleting information in the genetic code) to control the cells of the brain. Optogenetics has become very popular and is now being used in brain research laboratories all over the world. It is helping us to discover many new things about the brain. Here, we explain what makes optogenetics so special for studying the brain.

Special cells in the brain and nervous system, called neurons, work together to produce all of our thoughts and behaviors. In order to understand how the brain controls behavior, we need to understand how neurons communicate. The human brain is very complex, but many of the properties of the human 
ELECTRICAL

STIMULATION

\section{STUDY}

A technique for activating neurons or neural pathways by inserting a small electrode and sending an electrical current in to the tissue. This causes changes in the electrical activity of the tissue.

\section{NEUROSCIENCE}

A branch of science that studies the brain and nervous system. brain are similar to those of other animals. This means that neuroscientists (scientists who study the brain and nervous system) can use simple animals to discover new things about the human brain. This was how communication between neurons was discovered.

\section{HOW DO NEURONS COMMUNICATE WITH EACH OTHER?}

Neurons communicate using a combination of electrical and chemical activity. Early scientists discovered this through observations and clever experiments. In the late 1700s, an Italian scientist named Luigi Galvani was walking through a market during a lightning storm. He saw some frog legs for sale and noticed that they were twitching. He hypothesized that the electricity of the storm was activating the nerves in the frog legs. He decided to test this hypothesis in his laboratory. Galvani used an object that allows electrical current to flow, called an electrode, to pass an electrical current on to the frog nerve. This caused the frog leg to twitch. This was the first electrical stimulation study in neuroscience [1]. From this finding, Galvani concluded that neurons could use electrical signals to pass information. This is important to know! Now that we know how neurons talk to each other, we can start to speak their language. We can use electrical signals to turn some neurons on, and see what happens next. This is exactly what scientists started to do.

It wasn't until the 1930s that electrical stimulation was used to map the human brain. Dr. Wilder Penfield, a brain surgeon, was working with patients who had epilepsy. Epilepsy causes abnormal electrical signals in the brain and can be very dangerous. In extreme cases, brain surgery is required to stop the epilepsy. Dr. Penfield wanted to map the brains of his patients to find out which parts of the brain were most important. This would help him to know which brain areas he should not operate on. To map the brain, he used electrical stimulation, just as Galvani had. He lowered a small electrode into the motor (movement) areas of the brain. Then, he sent a small electrical signal and observed the patient's movements. Stimulation in one area of the brain caused a finger twitch, while stimulation in a slightly different area of the brain caused a foot twitch. This led Dr. Penfield to realize that certain areas of the brain control very specific areas of the body. Dr. Penfield noticed that the location of the motor areas of the brain was similar in all of his patients. He created diagrams of his results, which gave us the first functional map of the motor areas in the human brain [2]. Dr. Penfield's functional maps, known as the homunculus, are still used today.

Since the 1930s, brain stimulation experiments have changed. Electrical stimulation studies have some drawbacks. One problem is that the brain might be damaged when an electrode is inserted. Another problem is that electrical stimulation activates tissue in a very general, non-selective way (Figure 1A). 


\section{FIGURE 1}

A. In an electrical stimulation study, all cells close to the stimulation site will be activated.

B. Blue light does not normally affect neurons.

C. Blue light will selectively activate neurons with channelrhodopsin-2 (ChR2).

\section{OPTOGENETICS}

A technique that uses a combination of light and genetic engineering to control the activity of a cell.

\section{GENETIC}

\section{ENGINEERING}

The process of changing the information in the genetic code (the blueprints) of a living thing by adding or deleting information. Genetic engineering is sometimes called genetic modification.

\section{OPSINS}

Proteins that respond to a specific type of light (for example, ChR2 only responds to blue light). In neuroscience, these proteins are used to control neuron activity.

\section{CHANNELRHOD-} OPSIN-2 (CHR2)

An opsin that responds specifically to blue light. When ChR2 is inserted into neurons, blue light can be used to turn those neurons on. ChR2 is currently the most popular opsin for optogenetic studies.
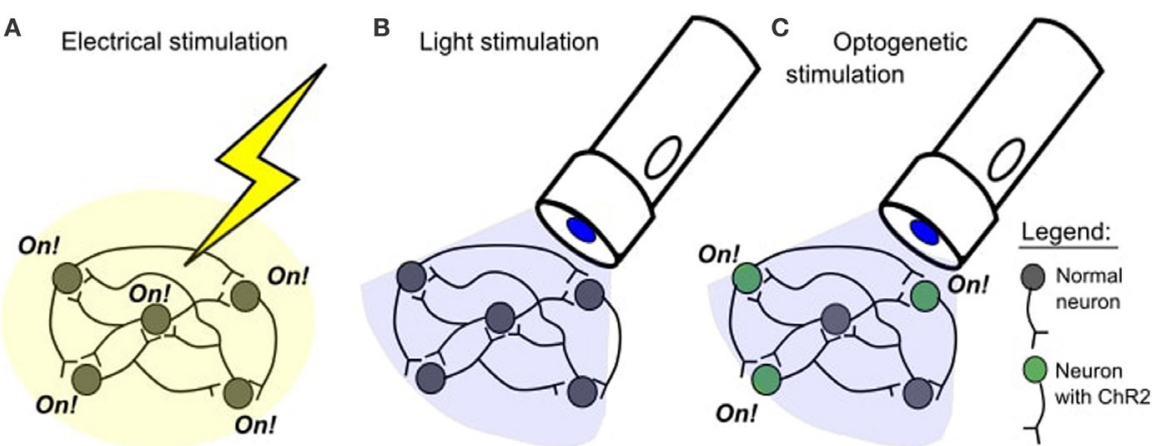

FIGURE 1

It is like using a bulldozer when a shovel will do-the bulldozer is effective, but it is not very precise or careful. In 2005, a new technique was created to allow for more precise brain stimulation. This technique is called optogenetics.

\section{WHAT IS OPTOGENETICS?}

Optogenetics is a method for controlling a neuron's activity using light and genetic engineering. Genetic engineering is a process where scientists change the information in the genetic code (the blueprints) of a living thing. In optogenetic studies, scientists take the genetic code of the neurons they want to study and add a new piece of code to it. The new code allows these neurons to make special proteins, called opsins, which respond to light. Opsins occur naturally and were first discovered in algae, which use these proteins to help them move toward light. But how does the opsin get into the neuron? This requires some specialized laboratory techniques. Let's look at a mouse as our example. To get the opsin into the neurons of a mouse, the genetic code for the opsin must be carefully inserted into the genetic code for the neurons in the mouse. If this is done correctly, now every neuron in the mouse should have the opsin. Because we understand a lot about the genetic code of the mouse, we can choose where to put the opsin. We can insert the code into a specific type of neuron, or into a specific location in the brain. We can choose exactly which neurons we want to control.

In neuroscience, the most popular opsin is called channelrhodopsin-2 (ChR2). This opsin comes from the green algae Chlamydomonas reinhardtii [3]. ChR2 is activated by blue light, meaning that it only works when blue light shines on it and it doesn't respond to other types of light. When ChR2 is inserted into neurons, it means that the neurons can be turned on with blue light. The neurons with ChR2 will only be on for as long as the blue light is shining on them [4]. This gives us precise control over the timing of the neuron's activity. Normally, neurons are not affected by blue light (Figure 1B), so only the neurons that have ChR2 will be affected by blue light (Figure 1C). 


\section{FIGURE 2}

A. Just like there are many roads in a city, there are many pathways in the brain. B. An electrical stimulation study is like sending all of the cars out on the roads at once.

C. An optogenetic stimulation study is like sending only one type of car out on the roads.

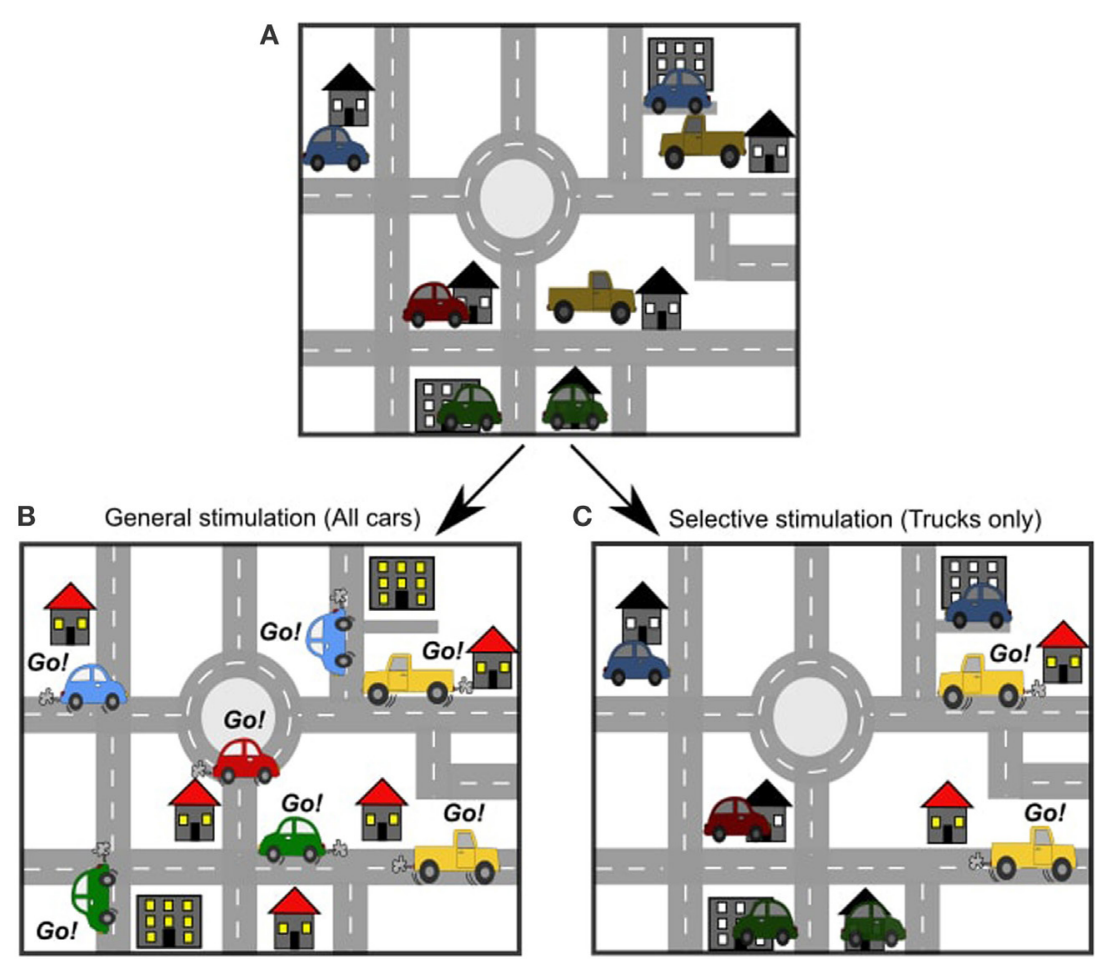

FIGURE 2

\section{OPTOGENETIC STIMULATION IS MORE SPECIFIC THAN ELECTRICAL STIMULATION}

Just like there are many roads in a city, there are many pathways in the brain. If we want to know how Point A connects to Point B in a city, we can simply look at all the roads and draw a road map. This is a type of structural map: it helps us to understand how the roads are set up. But, there are usually many ways to get from Point A to Point B, so how do we know which way is the most popular? To figure this out, we need to look at the cars driving on the roads as they go from Point A to Point B. This is a functional map: it helps us to understand how the roads are being used. In the brain, neurons are like the roads, and the signals that travel from neuron to neuron are like the cars. Normally, the brain is very active and there are lots of cars on the roads at all times. All over the brain map, cars are starting and stopping their journeys at different times. Because, there is so much activity, we cannot see any patterns or understand how things are related. To figure out the patterns, it would be useful to be able to control when and where the cars begin their journey.

Imagine that there are cars in every driveway in our city. These cars are waiting for a signal to go out on the roads (Figure 2A). In an electrical stimulation study, we can control when the cars start traveling, but we don't have a lot of control over which cars will go out on the roads. With electrical stimulation, the stimulation is general. All cars close to the stimulation will be sent on the road. This means there is a lot of activity to follow (Figure 2B). In an optogenetic stimulation study, we can choose exactly which cars we want to 
go out on the road and when. We can choose a group of cars by location (for example, we could choose to have all the cars in one neighborhood go out on the roads), or we can choose by type of car (for example, we could choose to have only trucks go out on the roads) (Figure 2C). This is selective stimulation. The movement of the cars is a lot easier to follow in this case. It tells us more about how specific cars operate on the roads.

\section{HOW IS OPTOGENETICS USED TO MAP THE BRAIN?}

Optogeneics can be used in several different ways for mapping the mouse brain (reviewed in Ref. [5]).

Just like we can zoom out on a road map of a city to see the major highways (Figure 3A), or zoom in on the map to see a single city block (Figure 3B), we can also zoom in or out on the brain. We can zoom out from the brain to see how large areas of the brain connect and work together (Figure 3C). This bigpicture view is good if we are interested in the way information travels over long distances in the brain, or which areas of the brain are connected to each other. For example, big cities tend to have more roads and highways passing through them, because lots of people travel to and from these cities. Using optogenetics to stimulate one brain area and record the responses in other brain areas, we can figure out which areas of the brain have the most traffic. This is important for understanding how certain behaviors are produced, but also might be important for understanding what happens if the brain is damaged in a particular area (for example, if there is an accident on fourth street, how will the traffic reroute?).

We can also zoom in on the brain to see how individual neurons connect (Figure 3D). Using optogenetics, we can investigate how the neurons work together, by using light to turn some neurons on and record the response of

\section{FIGURE 3}

A. Just like you can look at a whole city map, B. or zoom in on an individual road, you can also look at the connections in the whole brain C., or zoom in on a specific group of cells $\mathbf{D}$.

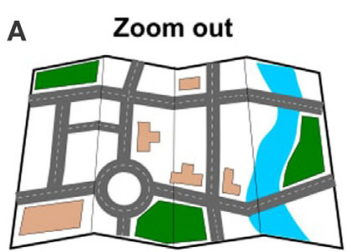

C
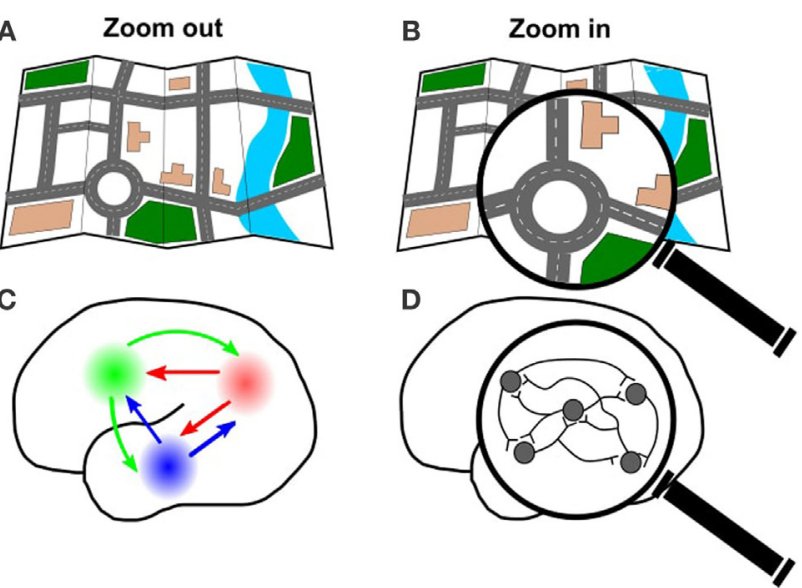

FIGURE 3 


\section{STROKE}

Normally, blood carries oxygen and other important nutrients to the brain. When the blood supply is disrupted or reduced, the brain doesn't get the things that it needs to function properly. This is called a stroke, and this can cause lasting problems and dysfunction.

the other neurons. This detailed view is useful for understanding how and when the neurons communicate with each other. This could be very useful for investigating diseases that disrupt communication between neurons in a particular area, which is what happens when someone has a stroke (read more about this in the next section).

Optogenetic mapping creates many possibilities for investigating how the brain works. As optogenetic techniques improve and more opsins are created or discovered, there is the possibility for even more control in brain stimulation studies. Maybe we will be able to use multiple opsins to control several different types of neurons at the same time. Because each opsin responds to a specific type of light, we could use different light to control different types of neurons. In fact, some opsins act to turn neurons off when the right type of light is present.

In our example of mapping cars in the city, we could use multiple signals to control the movement of the cars. We could have one set of cars go out on the road when we give one signal (say, blue light) and another set of cars go out on the road when we give a different signal (say, a red light). Using this setup, we could start experimenting with these two sets of cars: what happens if the red light cars go first? What happens if the blue light cars go first? What happens if they go at the same time? This would help us to understand how these different sets of cars interact with each other.

So how does a scientist choose which technique or which opsin to use? The answer will depend on the question the scientist wants to explore. The next section will highlight some of the questions that have been investigated using optogenetics.

\section{RECENT DISCOVERIES USING OPTOGENETICS}

Brain scientists began using optogenetics in 2005 [3]. Since then, optogenetic methods have been used to study the brain from many different points of view-from the communication of a cluster of individual neurons, to the interactions between large brain regions (reviewed in Ref. [5]). Many other studies have used optogenetic methods to investigate different topics and questions. Some recent questions are: where is fear in the brain? How is risk and reward calculated? How are memories stored? (reviewed in Ref. [6]). We used optogenetics in mice to investigate how the brain changes after a stroke [7]. A stroke happens when the blood supply to an area of the brain is disrupted or reduced. This is dangerous because the blood supply carries oxygen and other important nutrients that the brain needs to survive. If any area of the brain goes for too long without oxygen, the neurons in that area will eventually die. This causes problems for that particular area of the brain and for any other brain areas that are connected to it. In our study, we wanted to investigate how a small stroke to one area of the brain affected many other areas of the 
brain. To begin, we used ChR2 to help us draw a functional map of the mouse brain. We compared the maps between animals with a stroke and without a stroke. We found that the maps changed over time. At 1 week after stroke, the overall brain activity was very low. Surprisingly, activity was low even at an area far away from the stroke. By 8 weeks after stroke, the overall brain activity was higher, but not back to normal. From these data, we concluded that even a small stroke can have a big effect on how the brain works as a whole. Understanding what happens to the brain after a stroke could help scientists create better treatments for stroke patients. This is just one example of how useful optogenetics can be for investigating questions about the brain. It is likely that brain scientists will continue to use optogenetics for many years to come.

\section{ORIGINAL SOURCE ARTICLE}

Lim, D. H., LeDue, J., Mohajerani, M. H., Vanni, M. P., and Murphy, T. H. 2013. Optogenetic approaches for functional mouse brain mapping. Front. Neurosci. 7:54. doi:10.3389/fnins.2013.00054

\section{REFERENCES}

1. Kolb, B., Whishaw, I. Q., and Teskey, G. C. 2016. An Introduction to Brain and Behavior. 5th ed. New York, NY: Worth.

2. Penfield, W., and Edwin, B. 1937. Somatic motor and sensory representation in the cerebral cortex of man as studied by electrical stimulation. Brain 60(4):389-443. doi:10.1093/brain/60.4.389

3. Nagel, G., Szellas, T., Huhn, W., Kateriya, S., Adeishvili, N., Berthold, P., et al. 2003. Channelrhodopsin-2, a directly light-gated cation-selective membrane channel. Proc. Natl. Acad. Sci. U.S.A. 100:13940-5. doi:10.1073/pnas.1936192100

4. Boyden, E. S., Zhang, F., Bamberg, E., Nagel, G., and Deisseroth, K. 2005. Millisecond-timescale genetically targeted optical control of neural activity. Nat. Neurosci. 8:1263-8. doi:10.1038/nn1525

5. Lim, D. H., LeDue, J., Mohajerani, M. H., Vanni, M. P., and Murphy, T. H. 2013. Optogenetic approaches for functional mouse brain mapping. Front. Neurosci. 7:54. doi:10.3389/fnins.2013.00054

6. Deisseroth, K. 2015. Optogenetics: 10 years of microbial opsins in neuroscience. Nat. Neurosci. 18(9):1213-25. doi:10.1038/nn.4091

7. Lim, D. H., LeDue, J., Mohajerani, M. H., and Murphy, T. H. 2014. Optogenetic mapping after stroke reveals network-wide scaling of functional connections and heterogeneous recovery of the peri-infarct. J. Neurosci. 34(49):16455-66. doi:10.1523/JNEUROSCI.3384-14.2014

SUBMITTED: 03 February 2017; ACCEPTED: 31 August 2017;

PUBLISHED ONLINE: 20 September 2017.

EDITED BY: Daniel Ansari, University of Western Ontario, Canada 
CITATION: Lim DH, LeDue J (2017) What Is Optogenetics and How Can We Use It to Discover More About the Brain? Front. Young Minds 5:51. doi:10.3389/frym.2017.00051

CONFLICT OF INTEREST STATEMENT: The authors declare that the research was conducted in the absence of any commercial or financial relationships that could be construed as a potential conflict of interest.

COPYRIGHT (C) $2017 \mathrm{Lim}$ and LeDue. This is an open-access article distributed under the terms of the Creative Commons Attribution License (CC BY). The use, distribution or reproduction in other forums is permitted, provided the original author(s) or licensor are credited and that the original publication in this journal is cited, in accordance with accepted academic practice. No use, distribution or reproduction is permitted which does not comply with these terms.

\section{REVIEWED BY}

\section{PRUTHVI, 15 YEARS OLD}

Hi, my name is Pruthvi. I am 15 years old and I live in Ontario. I am in grade 10. I am an intrapersonal learner. In my free time I like to read which includes books, articles, and news. I like spending time with nature and I like to capture the moments with pictures. My favorite subject is science. My love for science developed in my last years of elementary school. I would like to pursue my future in the medical field.

\section{AUTHORS}

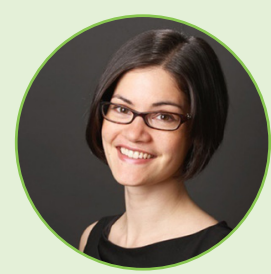

\section{DIANA H. LIM}

She completed her Ph.D. in Neuroscience at the University of British Columbia, where she used optogenetic mapping to study recovery after stroke. She thinks the brain is pretty cool, and currently teaches psychology and neuroscience at Concordia University, in Montreal, QC, Canada. She hopes to spread her love of the brain to her students. *diana.lim@ concordia.ca

\section{JEFFREY LEDUE}

He studied physics at Dalhousie University and the University of Victoria. Using his physics background he helps neuroscientists employ lasers and optics to understand the brain with optogenetics. He currently works at the Djavad Mowafaghian Centre for Brain Health at the University of British Columbia, in Vancouver, BC, Canada. 